\title{
DE L'UTOPIE VERS L'ÉDEN : LE JOUEUR DE FLÛTE DE LOUIS HAMELIN
}

Élise Lepage, Université de Colombie-Britannique

\section{Résumé analytique}

Dans Le Joueur de flûte (2001), Louis Hamelin suit les péripéties de son narrateur qui quitte son Québec natal pour se rendre sur une île de la côte ouest pour retrouver son père, écrivain disparu. En se fondant sur les catégories établies par le géographe Luc Bureau, cette réflexion s'attache à montrer comment l'île Mere est représentée comme un espace édénique qui reprend, en les parodiant, les stéréotypes couramment attachés à la côte Ouest. Il faut cependant prendre en compte la dimension paratextuelle de ce roman afin de rendre la complexité de son rapport à la spatialité - tant fictive que textuelle.

\section{$* * *$ \\ «Bon, O.K. Je m'appelle Ti-Luc Blouin et j'ai été conçu dans une commune de la côte ouest à la fin des} années soixante. » (JF 15) Ainsi commence Le Joueur de flûte de Louis Hamelin, paru en 2001. Ce roman suit les péripéties de Ti-Luc, le narrateur qui quitte Montréal pour retrouver son prétendu père, Forward Fuse, « obscur écrivain américain qui avait eu son heure de gloire à San Francisco au début des années soixante» et « inventeur du fuck writing » (JF 16) sur une île de la côte ouest du Canada. Cette quête du père aboutit étrangement sur « l'île Mere » où se situe l'essentiel de l'intrigue. Le microcosme de l'île se partage entre une bande de quasi «écoterroristes » (JF 50) et les Onani's, peuple autochtone, les deux populations luttant activement contre les projets de déboisement d'une puissante compagnie forestière. Oscillant entre ironie et bienveillance, Ti-Luc décrit ce monde qui semble s'être arrêté aux utopies des années 1970 et cherche à s'y insérer dans l'espoir de retrouver son père, surnommé tantôt le Joueur de flûte, tantôt Mister Big. C'est à Deep Point, au cœur de l'île, qu'il le rencontre, retrace son existence ravagée par les excès de la drogue et d'expérimentations pseudo-littéraires, et lui révèle son identité. Le lendemain, Mister Big se suicide et, « redevenu un étranger» (JF 188), Ti-Luc n'a plus qu'à quitter l'île et regagner le Québec.

Cette réflexion s'attache à montrer comment la représentation de l'île Mere, perçue comme un espace étranger, s'élabore dans le roman comme un espace édénique, reprenant - mais aussi parodiant - plusieurs clichés attachés aux représentations de la Colombie-Britannique. Espace édénique donc, à opposer à un espace utopique, qui serait celui du Québec, lieu d'origine de Ti-Luc. Espace édénique et espace utopique : ces deux modes d'appréhension de la spatialité sont empruntés à l'ouvrage Entre l'éden et l'utopie (1984) du géographe Luc Bureau. À quel point ces concepts géographiques permettent-ils d'appréhender l'espace fictif du roman de Hamelin ?

Nous identifierions ainsi en quoi la représentation de l'espace d'origine, le Québec, correspond à ce que Bureau caractérise comme un espace utopique que cherche à fuir le narrateur. Au rebours, tout en l'île indique une représentation de l'Éden, parfois jusqu'à la parodie. Mais finalement, un autre espace édénique peut être décelé, non plus au niveau de la mimesis, mais au niveau paratextuel. Nous tenterons alors de montrer en quoi ce roman peut se lire comme la tentation de Hamelin de créer un «Éden textuel », situé dans un nowhere entre monde fictif et monde référentiel. 


\section{Le lieu d'origine : un espace utopique}

Luc Bureau définit I'Utopie comme la tentation d'apprivoiser le cosmos (15). Ce mode d'organisation de

l'espace correspond selon lui au

domaine de la culture, de l'organisation, des forêts en quinconce, des champs rectangulaires et bien alignés, des maisons-machines-à-habiter de Le Corbusier, des routes imperturbablement droites [...]. L'utopiste n'est pas regardant sur les moyens; le bulldozer, le rouleau compresseur, la grosse calculatrice électronique et la lunette d'arpenteur sont ses instruments préférés. L'état social auquel il aspire est si parfait, qu'une fois achevé, les retouches deviendront inutiles et indésirables. (12)

Il montre comment cette conception utopique de l'espace a informé historiquement l'organisation de l'espace québécois traditionnel :

L'aventure européenne en terre d'Amérique sera d'abord et avant tout un exercice spéculatif cherchant à régir in abstracto le destin d'un continent [...]. Dès le premier instant, cette Amérique est un non-lieu, une terre que l'imaginaire invente et qu'il peuple de ses fantasmes. (109)

Or, ces fantasmes sont étroitement aiguillés par une rationalité toute cartésienne et une volonté d'uniformité. Ainsi naît le rang, unité rurale reproduite à l'échelle de la province, mais aussi la géométrie impeccable de Montréal, ville fortifiée et organisée telle un échiquier. Et Bureau de conclure que « l'utopie a enfin trouvé une rade où mouiller ses épaves » (109), avant de remarquer :

Avant I'Amérique et Samuel de Champlain, [...] la campagne [...]semblait être une lente émanation du sol [...]. Nul n'aurait pu dire la part exacte de la nature, des dieux et des hommes dans ses configurations mimétiques. Puis, un jour, tout bascula. La campagne n'était plus une sécrétion du sol, ni des dieux, ni de la tradition, ni du temps, ni du savoirfaire collectif; elle était devenue une équation mathématique. La seule raison d'être du sol était dorénavant de servir de support à cette équation sacrilège.

Sol désacralisé. Espace dramatisé. Génie des lieux assassinés. Savoir démythifié. Voilà l'immense drame qui se joue sur les rives du Saint-Laurent dans les années 1625-1660. Pour la première fois sur la terre des hommes, ce n'est plus un jardin, un monument, une rue ou une ville que l'on soumet à l'isotropie géométrique, mais la campagne actuelle et virtuelle de tout un continent. Car, dès 1660, si tout n'est pas géométrisé en terre laurentienne, tout est perçu comme géométrisable. (118-9)

Du XVII ${ }^{e}$ à la fin du XXe siècle, seules les apparences ont changé, mais le projet est bien toujours le même, celui d'un espace à maîtriser et à rationaliser. Planification, standardisation, ségrégation, transparence, géométrie, clôture et artificialité sont les maîtres-mots qui définissent les Utopies d'aujourd'hui, U-topies dont le nom indique que leur forme réalisée, accomplie, ne se trouve nulle part. Pourtant, I'Utopie est bien présente autour de nous, sous des formes plus ou moins édulcorées : villes-nouvelles, projets-champignons faisant en quelques mois surgir des dizaines de maisons similaires au milieu d'un champ, organisation des zones d'activités commerciales ou industrielles, des centres d'achat: partout le même idéal d'égalitarisme et d'optimisation de l'espace - celui que décrit déjà Bureau pour l'aménagement de ce qui deviendra le Québec -, cet idéal qui conduit aujourd'hui à des aménagements standardisés reproductibles à l'infini : «De Bangkok à Bahreïn à Bali à Brasilia à Bangui, le même terrain de golf à vingt-sept trous» (SG 295), note le narrateur du Soleil des gouffres, précédent roman de Hamelin. 
Cette conception utopique de l'espace est bien présente dans l'œuvre de Hamelin, perçu à ses débuts comme une voix de la "génération X" qui a grandi dans ce type d'habitat et de construction. La satire de I'Utopie se manifeste dès La Rage, son premier roman, où la ville de Laval est représentée comme le paradigme de la banlieue standardisée, « la banlieue-bidon [...] ordonnée comme un cimetière » (LR 308) : « Je suis de retour à Laval, Laval des avalés, Laval qu'on peut parcourir à l'envers ou à l'endroit, Laval dans tous les sens, Laval où j'ai vécu ma jeunesse de Jonas au fond du ventre d'un bungalow. Baleine sur le gril, banlieue-barbecue, la vie à petit feu. » (LR 304). Hamelin n'épargne pas ses facilités à créer des jeux de mots et des échos littéraires - à L'Avalée des avalés de Ducharme - pour stigmatiser la banlieue québécoise. D'ailleurs, tant La Rage, Cowboy, Betsi Larousse que Le Joueur de flûte peuvent se lire comme des tentatives des narrateurs d'échapper provisoirement aux mailles de I'utopie urbaine du Québec contemporain. Au début du Joueur de flûte, Ti-Luc retrace son bref parcours professionnel dans ce monde à quadriller, à cartographier : « À l'âge de vingt-cinq ans, sur la foi d'obscures études que j'étais censé poursuivre, je [...] fus catapulté chargé de projet en environnement. [...] Mon boulot consistait, en gros, à dresser la cartographie des sources de pollution touchant l'ensemble du territoire de l'île de Montréal. » (JF 24-5). Pourtant, c'est sur une autre carte qu'il se prend à rêver :

J'avais déplié la carte de la Colombie-Britannique sur le zinc. Entre deux gorgées de bière, je me laissais absorber par la contemplation de ces grands espaces miniaturisés, me demandant laquelle, parmi la myriade d'îles éparpillées qui dansait sous mes yeux dans la mauvaise lumière, était la terre qui m'intéressait. (JF 40)

Ti-Luc souhaite aller rencontrer son père sur Mere Island, petite île «tout au bout de la route», « en face de Virago, sur l'île de Vancouver » (JF 35). Derrière cette quête de l'origine paternelle, se profile également celle d'un monde autre que celui dans lequel il vit :

Pourquoi le moindre petit cueilleur de fruits québécois de l'Okanagan avait-il l'impression, encore aujourd'hui, de partir pour une contrée merveilleuse où la vie serait facile et abondante, où le lait coulerait des hautes terres et le miel des arbres ? Terre-Neuve n'était pas de taille à entretenir ce genre de rêveries. L'Utopie, depuis toujours, se trouvait à I'Ouest. (JF 38)

Si ce questionnement sur cette sorte de fascination persistante qu'exerce l'imaginaire de l'Ouest demeure pertinent, il faut cependant pointer que le narrateur se trompe de mot lorsqu'il dit que « I'Utopie [...] se trouvait à l'Ouest. » En effet, ses allusions au lait coulant de source ou au miel disponible sans effort rappellent bien davantage un monde édénique d'un naturel prodigue que les Utopies fondées sur des plans, des cartes et le labeur.

\section{L'Ouest ou la quête d'un espace édénique}

Nous l'avons souligné, le voyage de Ti-Luc vers l'île est une quête placée sous le signe de l'origine. Il s'agit initialement de retrouver l'origine paternelle certes, mais très vite le narrateur découvre un microcosme dont les habitants n'ont de cesse de maintenir, sinon de recréer un monde "originel", "naturel". Luc Bureau ne croit pas si bien écrire lorsqu'il caractérise l'Éden comme 
le pays de la nature originelle, des forces insoumises, des forêts excessives et indisciplinées, des huttes primitives se réclamant du modèle par excellence de la maison d'Adam au paradis, [...] des festivités spontanées et des amours bucoliques. C'est le royaume rêvé des écologistes enragés, des communautés beatnicks et des amateurs de camping sauvage. (12)

Le roman de Hamelin semble fournir une parfaite illustration de l'Éden ainsi conçu. Les premières pages de l'arrivée du narrateur sur l'île plantent rapidement un décor caractéristique : « Un Buswolks bariolé était garé près du bord, présence incongrue en l'absence de toute route. Nous avons accosté près d'un type en slip orangé figé dans une extase taï chi. » (JF 57). Dans ce monde peuplé d'adeptes du « rebirth » (68), de « strip poésie » (64), on discute très sérieusement des théories de l'«éro-communisme» (60) autour de différents « menus: végétarien, végétalien, lacto-végétarien et ichtyo-végétarien [...], la viande rouge éta[nt] complètement hors sujet » (61). Le catalogage des attributs essentiels du parfait beatnick est ainsi un peu trop exhaustif pour ne pas être perçu comme une caricature qui prête à sourire. Hamelin s'ingénie à reprendre tous les clichés et stéréotypes attachés aux îles isolées de la côte ouest en les parodiant, produisant des personnages et un décor hauts en couleurs, tout ceci sous le signe de l'humour et de la distanciation. Si les utopistes ont souvent considéré l'île comme un espace idéal où mettre en place leur projet, l'île du Joueur de flûte se présente bien moins comme une Utopie que comme un Éden dans la mesure où ses habitants cherchent à recréer un état d'harmonie supposé originel, où I'homme vivrait en parfaite osmose avec la nature qui l'entourerait.

Le choix de situer l'intrigue du roman dans une île n'est en effet pas indifférent. L'espace insulaire, coupé par la mer du reste du continent, invite à un détachement spatial. « Les chercheurs de paradis tendent à s'isoler, note Bureau, dans la campagne, la forêt, le désert ou... leur chambre à coucher » (49). « L'Éden est d'abord et avant tout un paysage intérieur où l'on ne parvient que par les sentiers du cœur : il faut se fermer les yeux et la raison pour l'apercevoir. » (Bureau 49) La quête de Ti-Luc le conduit à faire l'expérience de cette quête intérieure. Significativement, cette expérience se produit dans des lieux isolés et relativement clos qui fonctionnent comme des mises en abyme de l'espace insulaire. Son trajet jusqu'au cœur bien nommé de l'île, Deep Point, est l'occasion de ce détachement. Mais le processus est encore plus sensible dans les passages où Ti-Luc se réfugie dans une pruche géante qui lui sert de refuge à plusieurs reprises :

J'ai repéré une faille à la base d'un arbre et je m'y suis glissé. Alors, le temps et l'espace s'arrêtèrent. Je n'entendais rien d'autre que le bruissement de mon propre sang. L'arbre était une pruche de l'Ouest qui avait été un jour frappée par la foudre. Elle se dressait, évidée, et dans ses hauteurs, des rameaux continuaient d'arborer des touffes d'aiguilles vertes. Son cœur était devenu un tunnel d'une soixantaine de mètres menant de la terre au ciel. (JF 103, je souligne)

Tout un réseau sémantique lié à l'évocation de cet arbre creux indique le fait de se blottir, de se tapir qui rappelle les termes que relevait Bachelard dans la Poétique de l'espace (1957) dans ses réflexions sur la maison comme hutte. Ti-Luc devient ainsi un squatter à tous les sens du terme. Bureau rappelle ainsi que «to squat signifie: s'accroupir (position du fœtus), se blottir, s'aplatir, s'incorporer, se fondre et se confondre [... tandis que] le 
substantif squat désigne entre autres le repaire ou le refuge de certaines bêtes sauvages » (Bureau 75). Or, telle est bien la position que Ti-Luc adopte dans son repaire, « recroquevillé dans [s]on antre » (JF 210). Mais Ti-Luc est aussi un squatter au sens où il s'associe à la cause de défense des arbres des occupants de l'île puisqu'à la fin du récit, il refuse de quitter sa pruche encerclée par des policiers qui finissent par faire abattre l'arbre alors que le protagoniste est monté à son sommet, de l'intérieur. Ti-Luc est donc un squatter dans les deux sens du terme, entre repli physique - celui de la position fœtale - et repli idéologique - le refus de quitter l'endroit où il se réfugie : «Symboliquement, le squatter est donc celui qui s'accroupit au sol, s'y blottit, s'y incorpore, et refuse d'en être chassé. Puisqu'il fait corps avec la terre, puisqu'il en est le complément naturel, ses droits d'occupation sont censément les plus authentiques et incontestables qui soient », souligne Bureau (75), reprenant le discours de légitimation qui lie les deux sens du terme "squatter".

Tout incline à montrer que la représentation de l'île, et plus encore sa mise en abyme par Deep Point et l'arbre creux, fonctionnent comme des représentations de l'Éden, conception de l'espace reposant sur le modèle d'une nature fixée hors du temps et convoquant un imaginaire de l'origine non pervertie, de la nature à l'état sauvage, du ventre maternel, bref : du Paradis perdu. L'île s'oppose en cela en tout point à l'espace d'origine de TiLuc, marqué par la raison et ses quadrillages. Pourtant, si l'île et ses mises en abyme fonctionnent comme des représentations de l'Éden, un autre Éden, ne reposant pas sur le pacte mimétique romanesque, apparaît dans cette œuvre. En effet, si l'Éden est un espace de l'origine, mais aussi un espace impossible dans la mesure où il vise à arrêter le temps et à maintenir un équilibre prétendument originel, il faut alors le déceler dans un autre espace du texte, soit ses marges paratextuelles...

\section{Un Éden entre monde romanesque et monde référentiel ?}

Entre la dernière page du roman et la table des chapitres, s'insèrent en effet cinq pages intitulées «L'île Mere : une brève bibliographie commentée » dont une note précise que «les titres sont livrés dans le désordre » (JF 223). Le roman se referme donc sur cette bibliographie dont les références sont agrémentées d'un commentaire de Hamelin, allant de deux mots - «Sans commentaire» (JF 225), pour le livre intitulé Fuck Writing de Forward Fuse - à quelques paragraphes plus fournis pour d'autres. Remarquons notamment que le versant écologique de l'intrigue romanesque se résout dans le commentaire suivant la première référence, procédé qui opère un brouillage entre monde fictif et monde référentiel. Mais ce brouillage s'accentue pour le lecteur lorsqu'il découvre que plusieurs des auteurs des œuvres listées sont des personnages du roman. Cependant, vérifications faites, plusieurs de ces références dont les éditeurs et le lieu de publication sont dûment mentionnés, n'existent pas! Tel par exemple cet ouvrage du chef Art Watt, personnage du roman, qui aurait écrit un Totem et Tambour - titre qui rappelle étrangement le Totem et tabou de Freud. Ou encore, la première référence bibliographique dans laquelle Hamelin dénoue l'intrigue liée au destin de l'île. On ne peut manquer de noter le plaisir que l'auteur semble prendre 
à agrémenter son commentaire de formulations censées accentuer la vraisemblance de ce livre qui pourtant n'existe pas plus que sa prétendue maison d'édition :

La traduction française de cet ouvrage d'abord paru à Vancouver a été assurée, chez nous, par Renoir Mergini, de la sympathique petite maison d'édition du même nom. La version originale a vu le jour environ un an après l'arrêté historique de la Cour d'appel de la Colombie-Britannique, favorable aux Indiens onani's. Les juges, on le sait, statuèrent que la compagnie forestière ne pouvait, sous peine de créer un fait accompli, récolter les arbres de l'île Mere [...] (JF 223) (Nous soulignons).

Autrement dit, Hamelin reprend le procédé borgésien des textes apocryphes et met à profit l'autorité et le sérieux dont jouit traditionnellement le genre bibliographique pour estomper la frontière entre fiction et référentialité. Ce paratexte du Joueur de flûte crée donc un espace interstitiel, impossible, entre le monde fictif mis en place par le roman et le monde littéraire tel qu'il existe dans l'espace référentiel. Hamelin semble rêver, postuler un roman à la croisée entre la fiction et le monde littéraire en tant qu'institution, un roman dont l'intrigue trouverait son dénouement hors des limites de la fiction : dans la bibliographie, et notamment à travers les livres répertoriés dans cette bibliographie. L'insertion d'un schéma de l'Île Mere au tout début du roman participe également de ce brouillage entre espace fictif et référentiel. Le fait de représenter graphiquement l'île double sa représentation littéraire à venir et tend à accréditer son existence. Le schéma intègre d'ailleurs des éléments géographiques réels tels que l'océan Pacifique ou la route transcanadienne. Tout est fait pour rendre cette île la plus vraisemblable possible; il n'est pas jusqu'au nom du village de «Virago » qui rappelle quelque peu celui de « Tofino », potentiel référent. Le choix du titre fournit enfin un ultime indice de ce brouillage entre le monde fictif du roman et l'espace littéraire référentiel. En effet, l'auteur fait côtoyer en couverture son patronyme avec le titre du conte retranscrit par les frères Grimm, créant un jeu intertextuel qui amalgame un nom appartenant au monde référentiel - Hamelin - et un conte bien connu - Le Joueur de flûte de Hamelin. Or, chacun sait que le monde du conte est celui d'un espace imaginaire au temps immuable et en cela, quelque peu semblable aux représentations archétypales de l'Éden.

Il semblerait donc que l'Éden, dans ce roman de Hamelin, se situe donc autant au niveau de la mimesis qu'au niveau paratextuel. Si l'espace représenté - l'île Mere et plus encore Deep Point et la pruche creuse fonctionnent comme des représentations de l'Éden, il faut aussi compter avec le titre du roman et son intrigante «bibliographie » qui élabore ce qu'on pourrait désigner par l'expression d'«Éden textuel ». En effet, cette bibliographie présente au moins deux points communs fondamentaux avec les représentations de l'Éden telles que décrites par Luc Bureau. L'Éden, nous l'avons vu, est la quête du monde à son origine. Or, tant le titre du roman que sa bibliographie indiquent un questionnement portant sur l'origine : celle du patronyme de l'auteur, mais aussi celle des prétendues sources du roman. Adoptant un modèle tabulaire, la bibliographie s'offre comme une sorte d'arbre généalogique de l'œuvre, mentionnant ses influences, ses sources, les œuvres parentes ou cousines traitant de sujets connexes. Or, dans le cas du Joueur de flûte, ces origines bibliographiques sont toutes des sources plus ou moins fiables, inventées, rêvées par Hamelin pour donner assise à son roman. Nous avons de plus 
rappelé que l'Éden ne peut être « sauvegardé » que dans des aires restreintes et isolées de préférence (telle l'île). Les Édens se situent donc souvent dans des espaces en retrait de la civilisation et de sa temporalité. De la même façon, l'«Éden textuel » que constitue cette bibliographie se situe dans un espace marginal du texte, le paratexte. Elle est donc coupée du récit, rejetée dans un nowhere du livre qui relève pourtant encore du discours auctorial. Elle semble chercher sa place dans cet espace limite entre monde romanesque et monde référentiel. Tant donc par son isolement spatial au sein du livre que par sa fonction - répertorier les « origines » du roman, la bibliographie du Joueur de flûte fonctionne comme un Éden textuel problématique qui ne manque pas d'interroger les frontières communément admises entre fiction et référentialité, texte et paratexte.

Le Joueur de flûte met en place un imaginaire géographique opposant l'espace utopique du Québec d'où est originaire le narrateur et l'espace édénique d'une île de la côte ouest. Le premier est marqué par son organisation rationalisée, conceptualisée et cartographiée, lorsque le second croit protéger une parcelle de nature censée demeurée « originelle», authentique. En cela, Hamelin reprend non sans humour les stéréotypes couramment attachés à l'imaginaire de la côte ouest pour mieux les parodier et interroger la résistance de cet imaginaire. Si le caractère édénique de l'Île Mere peut surprendre - l'île étant traditionnellement l'emplacement rêvé des utopistes - les deux catégories que distingue Luc Bureau invitent à repenser ce présupposé.

Comme bien d'autres des romans de Hamelin, celui-ci peut se lire comme la tentative et tentation du narrateur de se soustraire à des espaces utopiques au profit de micro-édens excentrés. Mais Ti-Luc, pas plus que les précédents narrateurs du romancier, ne peut rester dans l'île édénique qui, d'une certaine façon, se révèle toute aussi «artificielle » que son pendant utopique. L'Éden repose sur des postulats, des désirs différents, mais à leur façon tout aussi rigides et irréalisables que la rationalité parfaite visée dans les projets utopiques. Le grand intérêt, selon nous, de ce roman est qu'il donne l'impression que Hamelin cherche à prolonger le rêve d'un de ces microespaces retranchés de l'ordre du monde, en le soustrayant également à l'ordre de la littérature et de la critique littéraire qui délimitent parfois arbitrairement ce qui est fiction et ce qui ne l'est pas, ce qui est texte ou paratexte. Tout comme I'Éden ou l'Utopie visent à remettre en question les modes de fonctionnement habituels de l'espace, l'Éden textuel auquel invite à penser Louis Hamelin remet en cause les conventions romanesques et amène malicieusement le dénouement de l'intrigue.

http://www.brocku.ca/cfra/voixplurielles06-01/index.html 


\section{Bibliographie}

Bureau, Luc. Entre l'éden et l'utopie. Montréal : Québec / Amérique, 1984.

Hamelin, Louis. La Rage. [1989]. Montréal : XYZ Éditeur, Coll. « Romanichels Poche », 1995.

---, Cowboy. [1992]. Montréal : XYZ Éditeur, Coll. « Romanichels Poche », 1998.

---, Betsi Larousse ou l'ineffable eccéité de la loutre. [1994]. Dossier de Julie Roberge. Montréal : XYZ Éditeur, Coll. « Romanichels Plus », 2002.

---, Le Joueur de flûte. Montréal : Boréal, 2001.

---, Le Soleil des gouffres. Montréal : Boréal, 1996.

' Sur la notion de paratexte, voir l'ouvrage de Gérard Genette, Seuils, Paris: Seuil, coll. « Poétique », 1987. 